\title{
Robertsonian chromosomes and the nuclear architecture of mouse meiotic prophase spermatocytes
}

\author{
Soledad Berríos ${ }^{1 *}$, Catalina Manieu, Julio López-Fenner², Eliana Ayarza', Jesús Page³, Marisel González',
} Marcia Manterola ${ }^{1}$ and Raúl Fernández-Donoso ${ }^{1}$

\begin{abstract}
Background: The nuclear architecture of meiotic prophase spermatocytes is based on higher-order patterns of spatial associations among chromosomal domains from different bivalents. The meiotic nuclear architecture depends on the chromosome characteristics and consequently is prone to modification by chromosomal rearrangements. In this work, we consider Mus domesticus spermatocytes with diploid chromosome number $2 n=40$, all telocentric, and investigate a possible modification of the ancestral nuclear architecture due to the emergence of derived $\mathrm{Rb}$ chromosomes, which may be present in the homozygous or heterozygous condition.

Results: In the $2 n=40$ spermatocyte nuclei random associations mediated by pericentromeric heterochromatin among the 19 telocentric bivalents ocurr at the nuclear periphery. The observed frequency of associations among them, made distinguishable by specific probes and FISH, seems to be the same for pairs that may or may not form $\mathrm{Rb}$ chromosomes. In the homozygote $\mathrm{Rb} 2 \mathrm{n}=24$ spermatocytes, associations also mediated by pericentromeric heterochromatin occur mainly between the three telocentric or the eight metacentric bivalents themselves. In heterozygote $\mathrm{Rb} 2 \mathrm{n}=32$ spermatocytes all heterochromatin is localized at the nuclear periphery, yet associations are mainly observed among the three telocentric bivalents and between the asynaptic axes of the trivalents.

Conclusions: The Rb chromosomes pose sharp restrictions for interactions in the $2 n=24$ and $2 n=32$ spermatocytes, as compared to the ample possibilities for interactions between bivalents in the $2 n=40$ spermatocytes. Undoubtedly the emergence of Rb chromosomes changes the ancestral nuclear architecture of $2 n=40$ spermatocytes since they establish new types of interactions among chromosomal domains, particularly through centromeric and heterochromatic regions at the nuclear periphery among telocentric and at the nuclear center among Rb metacentric ones.
\end{abstract}

Keywords: Robertsonian chromosomes, Mouse spermatocytes, Bivalent associations, Nuclear architecture

\section{Background}

During the meiotic prophase of spermatocytes, chromosomes form a distinctive arrangement in the nuclear space, governed mainly by the synapses between homologous chromosomes that form bivalents and by the union of their ends to the nuclear envelope [1-3]. The bivalent configuration generates a meiotic nuclear architecture dependent on the chromosome characteristics [4-6]. Consequently,

\footnotetext{
* Correspondence: sberrios@med.uchile.cl

'Programa Genética Humana, ICBM, Facultad de Medicina, Universidad de Chile, Santiago, Chile

Full list of author information is available at the end of the article
}

this architecture is subject to modification by chromosomal rearrangements.

In this work, we investigate how the ancestral nuclear architecture of Mus domesticus spermatocytes with a diploid chromosome number $2 n=40$, all of them telocentric, is modified by the emergence of derived $\mathrm{Rb}$ metacentric chromosomes, which may be present in the homozygous or heterozygous condition.

Synapsis between homologous chromosomes is a process that culminates with the formation of the synaptonemal complex (SC). The SC is a tripartite proteinaceous scaffold consisting of two lateral elements (homologous chromosome axes) and a medial component that stabilizes the

\section{Ciomed Central}

(C) 2014 Berrios et al.; licensee BioMed Central Ltd. This is an Open Access article distributed under the terms of the Creative Commons Attribution License (http://creativecommons.org/licenses/by/4.0), which permits unrestricted use, distribution, and reproduction in any medium, provided the original work is properly credited. The Creative Commons Public Domain Dedication waiver (http://creativecommons.org/publicdomain/zero/1.0/) applies to the data made available in this article, unless otherwise stated. 
joint between the homologous chromosomes forming the bivalents [7-10].

Numerous proteins are involved in the SC structure. SYCP3 protein is the main constituent of the axis of each homologous chromosome, and SYCP1 protein is located between the axes of homologous chromosomes, binding them together in synapsis with transverse filaments [11,12]. Both ends of each bivalent SC are attached to the nuclear envelope, so that each SC forms an arc of different extension depending on the length of the synapsed chromosomes. The chromatin, organized in loops, is connected to the lateral elements of the SC, hence the chromatin domains are sequentially ordered along each bivalent SC. Finally, the SC'trajectory determines the place for the chromosomal domains to occupy within the nuclear space. This is not trivial, because interactions or associations among heterologous chromosomal domains depend on the real possibility of establishing contacts between them together with their structural and functional affinities that could favor the consolidation of such interactions.

In this regard, the spermatocytes of Mus domesticus $2 n=40$ containing 19 autosomal telocentric bivalents with abundant pericentromeric heterochromatin near their proximal ends naturally favor the associations among them over the nuclear envelope [4]. Comparative analysis of the observed combinations of associated and non-associated bivalents in the spermatocytes, and the predictions of an ad-hoc developed probabilistic model for associations between indistinguishable elements, suggest that these associations could indeed take place randomly [6]. Notice that associations are not a phenomenon unique to meiotic prophase cells. They have also been described in Mus somatic cells with highly complex chromocenters, involving several chromosomes and the nucleoli [13].

Large blocks of satellite DNA (pericentromeric heterochromatin) surround the centromere and extend towards the proximal end of each chromosome, thus favoring the occurrence of $\mathrm{Rb}$ translocations in Mus. Double-strand breaks of DNA with nearly identical base components, in addition to physical proximity, facilitates the fusion of different chromosomes [14]. Rb translocations involve double-strand DNA breaking at the centromere level in two telocentric (acrocentric) chromosomes, followed by a repair (fusion) that binds the respective long arms, creating a metacentric $\mathrm{Rb}$ chromosome. The short arms ( $\mathrm{p}$ ) of the original telocentric chromosomes, including the proximal telomeres, part of the satellite DNA, and generally one centromere, are all lost. However, this loss of DNA does not significantly alter the total amount of DNA as compared with the standard Mus karyotype [15]. Rb translocation is the most common chromosomal rearrangement in mammals [16] and represents the type of chromosomal change that most effectively contributes to differentiation or speciation of natural populations [17].

In $M u s, \mathrm{Rb}$ translocations have resulted in more than 40 different chromosomal races (or subspecies), ranging from $2 n=40$ to $2 n=22$. These chromosomal races are natural populations characterized by altogether about 100 Rbs chromosomes with different combinations of arms [18], many of which emerged and spread extremely rapidly within populations of the standard karyotype [19].

Metacentric $\mathrm{Rb}$ chromosomes can become numerous in the Mus genome, leading to a reduction of ancestral telocentric chromosomes and to an emergence of new mixed karyotypes. Furthermore, crossing between wild homozygotes $2 \mathrm{n}=40$ and $\mathrm{Rb}$ homozygotes produce $\mathrm{F} 1$ hybrids in whose genomes the ancestral telocentric chromosomes are reunited with the metacentric derivatives. Trivalents are formed in the meiotic prophase spermatocytes of these mice, in which a metacentric chromosome is synapsed with the long arms of two telocentric chromosomes. Varying degrees of synapses are established among the proximal ends of the involved telocentric chromosomes, despite being heterologous [20].

The presence of Robertsonian ( $\mathrm{Rb}$ ) metacentric chromosomes, which can be multiple in this species, constitutes a valuable opportunity to study how these new chromosomes modify the original, ancestral nuclear architecture built just for telocentric bivalents.

To this end, we studied the meiotic nuclear organization of:

- Homozygote $2 \mathrm{n}=24$ spermatocytes, with 8 pairs of metacentric Rb chromosomes, 3 pairs of telocentric chromosomes, and the XY sex pair.

- Heterozygote $2 \mathrm{n}=32$ spermatocytes, with $8 \mathrm{Rb}$ metacentric chromosomes, 22 telocentric chromosomes, and the XY pair.

We further compared both families of spermatocytes with the nuclear architecture of the ancestral homozygote $2 \mathrm{n}=40$.

We found different patterns of nuclear architecture according to chromosome constitution of the spermatocytes. The $\mathrm{Rb}$ chromosomes present in $2 \mathrm{n}=24$ and $2 \mathrm{n}=32$ spermatocytes drastically restrict the possibilities for interaction between the heterochromatic domains as compared with the wealth of random associations observed in $2 n=40$ spermatocytes.

\section{Results}

Early prophase and the chromosome nuclear distribution in $\mathbf{2 n}=\mathbf{4 0}$ and $2 \mathrm{n}=\mathbf{2 4}$ spermatocytes

In early prophase nuclei, chromosome/bivalents are aggregated in a configuration known as the bouquet, in which the telomeric regions of all chromosomes cluster 
over a small area of the nuclear envelope. In this meiotic stage, the $2 n=40$ spermatocytes showed centromeres aggregated in one or two main groups at the nuclear periphery, while the respective axes or SCs described arcs toward the nuclear space (Figure 1a \& a'). In the bouquet configuration of the $2 \mathrm{n}=24$ spermatocytes, the centromeres appeared in two major clusters: one at the nuclear periphery, grouping the centromeres of the telocentric bivalents, and the other one toward the nucleus center, grouping the centromeres of the metacentric $\mathrm{Rb}$ bivalents (Figure $1 \mathrm{~b} \& \mathrm{~b}^{\prime}$ ).

When the early prophase nuclei were treated with DAPI that stains DNA rich in AT sequences, which in Mus is the DNA that underlies the pericentromeric heterochromatin, the $2 n=40$ spermatocyte nucleus showed one large DAPI-positive chromocenter whose location matched the regions of aggregated centromere. The $2 \mathrm{n}=24$ spermatocytes showed at least two DAPIpositive chromocenters, one at the nuclear periphery and the other toward the center of the nucleus. We showed previously that the initial aggregation of centromeres occurring in lepto-zygotene of $2 n=40$ spermatocytes will eventually resolve into several smaller aggregates that we can observe in pachytene or more advanced stages of meiotic prophase [6].

\section{Bivalent configuration and associations in $2 n=40$ pachytene spermatocytes}

In these meiotic nuclei all the telocentric SCs described regular arcs with the centromeres located in one extreme near the nuclear envelope. Figure 2a shows a wellpreserved pachytene nucleus with this chromosomal configuration. The focal plane is over the $\mathrm{SC}$ of one of the smallest bivalent where the centromeric domain close to the nuclear limit can be distinguished (Figure 2a). The abundant pericentromeric heterochromatin in all the bivalents preserving associations among them, produce chromocenters of different sizes, scattered throughout a

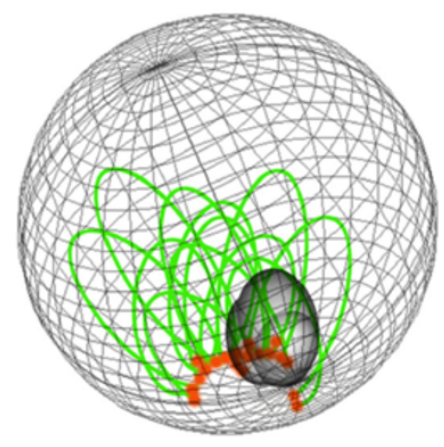

b

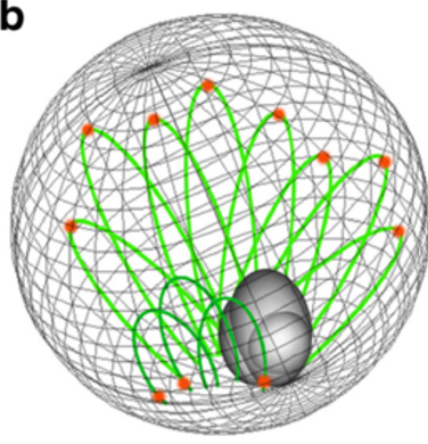

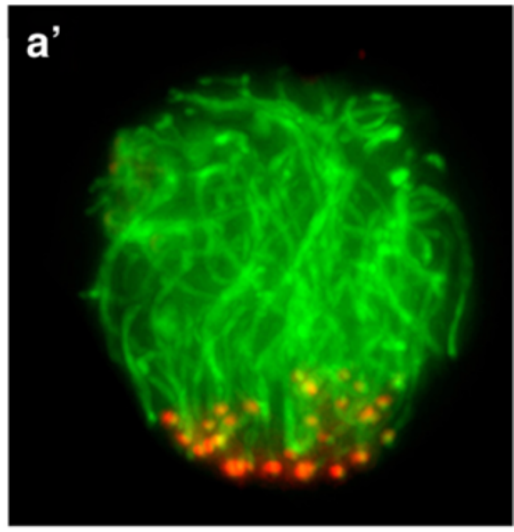

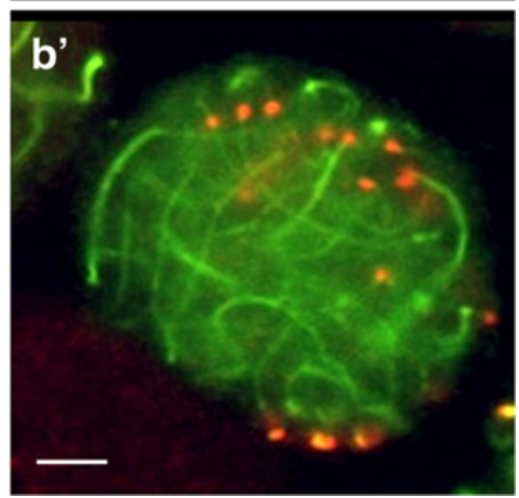

Figure 1 Bouquet configuration in early meiotic prophase nuclei of $2 n=40$ and $2 n=24$ spermatocytes. Diagram (a) and nucleus (a') showing the configuration of autosomal chromosomes in the "bouquet" of a $2 n=40$ spermatocytes. The chromatin of the sex bivalent is also represented (a). The centromeres (red) aggregate in one main group at the nuclear periphery, while the chromosomal axes (green) describe arches toward the nuclear space. Diagram (b) and nucleus (b') showing the configuration of autosomal chromosomes in the "bouquet" of a $2 n=24$ spermatocyte. The chromatin of the sex bivalent is also represented (b). The centromeres appear in two major clusters: one at the nuclear periphery, grouping the centromeres of the telocentric bivalents, and the other toward the nucleus center, grouping the centromeres of the metacentric Rb bivalents. The axes that emerge from the peripheral centromeres are shorter than those linked to the central centromeres. The chromosomal axes (green) and centromeres (red) in nuclei with preserved sphericity from $2 n=40$ and $2 n=24$ spermatocytes were identified by immunochemistry using, respectively, anti-SYCP3 and anti-CENPA antibodies. Bar $=5 \mu \mathrm{m}$. 

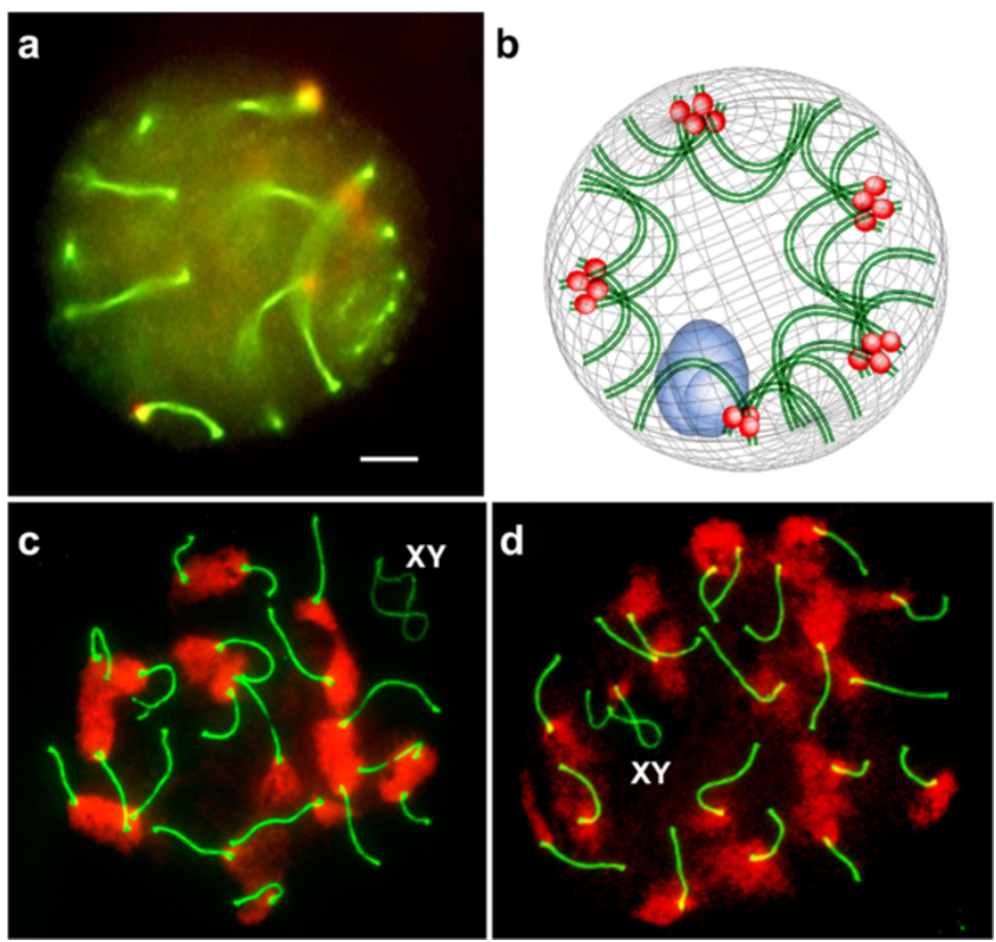

Figure 2 Nuclear architecture of spermatocytes $\mathbf{2 n = 4 0}$. a Distribution of telocentric bivalents in a 3D pachytene nucleus from $2 n=40$ spermatocytes. The synaptonemal complexes (green) and centromeres (red) were identified by immunochemistry using anti-SYCP3 and anti-CENPA antibodies. The 19 autosomal bivalents form regular arcs with the centromeres (red) (and the heterochromatin) in one extreme of each and consequently localized at the nuclear periphery. The focus is over one telocentric bivalent. Bar $=5 \mu \mathrm{m}$. b Scheme representing the nuclear architecture of $2 n=40$ spermatocytes. The centromeric regions (red) of all telocentric bivalents are located at the nuclear periphery. The bivalentes can be alone or associated in different numbers. The XY chromatin is also represented (blue). c \& d. Associations between telocentric bivalents through heterochromatin in $2 n=40$ spermatocytes. The SC (green) and the pericentromeric heterochromatin (red) were identified by immunochemistry using, respectively, anti-SYCP3 and anti-H3K9me3 antibodies. XY = sex bivalent. The19 autosomal bivalents appear singly or associated in different numerical combinations per spermatocyte. The combinatory presents in the nucleus of figure c) is: 5-4-3-2-2-2-1 = 19; and in the figure d) is: 6-3-2-2-2-1-1-1-1=19.

the nuclear envelope (Figure 1b). Therefore, each chromocenter is corresponds to a cluster of pericentromeric heterochromatin formed by the joint contribution of all those bivalents that remain associated between them throughout the prophase. The size of the chromocenter was proportional to the number of the associated bivalents. In pachytene nuclear spreads the clusters of heterochromatin were well preserved. By immunochemistry, both the axial elements of the SC as well as the pericentromeric heterochromatin, could be identified and, consequently, the number of bivalents participating in each chromocenter could be determined (Figure 2c \& d). The associations among chromosomes were studied in 100 microspreads of $2 n=40$ pachytene spermatocytes, considering the 19 autosomal bivalents as indistinguishable elements from one another. Each nucleus exhibited different combinations of chromosomal clusters. More than $60 \%$ of the pachytene spermatocytes presented groups of 4 , 5 or 6 bivalents, as the highest number of associated bivalents (Figure 2c \& d). Spermatocytes with no associations among bivalents or forming a cluster involving more than 11 associated bivalents were never observed (See [6]). Besides, we also studied the associative behavior of specific bivalents, identifying by FISH and chromosomal probes two bivalents at a time in 100 spermatocytes each (Figure 3). The probes were specific for chromosomes $9,14,16$, and 17 , and they were chosen because the chromosome pairs $9 / 14$ and $16 / 17$ form $\mathrm{Rb}$ metacentric chromosomes. In approximately $60 \%$ of spermatocytes, the two marked bivalents were found to be associated with other bivalents in a number of combinations, but not among themselves (Figure 3a \& b). In approximately 30 to $32 \%$ of the spermatocytes, the identified bivalents were alone, not associated with each other or with other bivalents. In about $8 \%$ to $11 \%$ of the studied spermatocytes, the two identified bivalents were associated to each other (Figure 3c). No differences was observed in the frequency of association between pairs of chromosomes that may form $\mathrm{Rb}$ metacentric chromosomes $(9 / 14,16 / 17)$, as compared to those pairs that do not form $\mathrm{Rb}$ chromosomes (9/17, 14/16) were observed (See Table 1). A computer simulation of all possible associations between the 19 

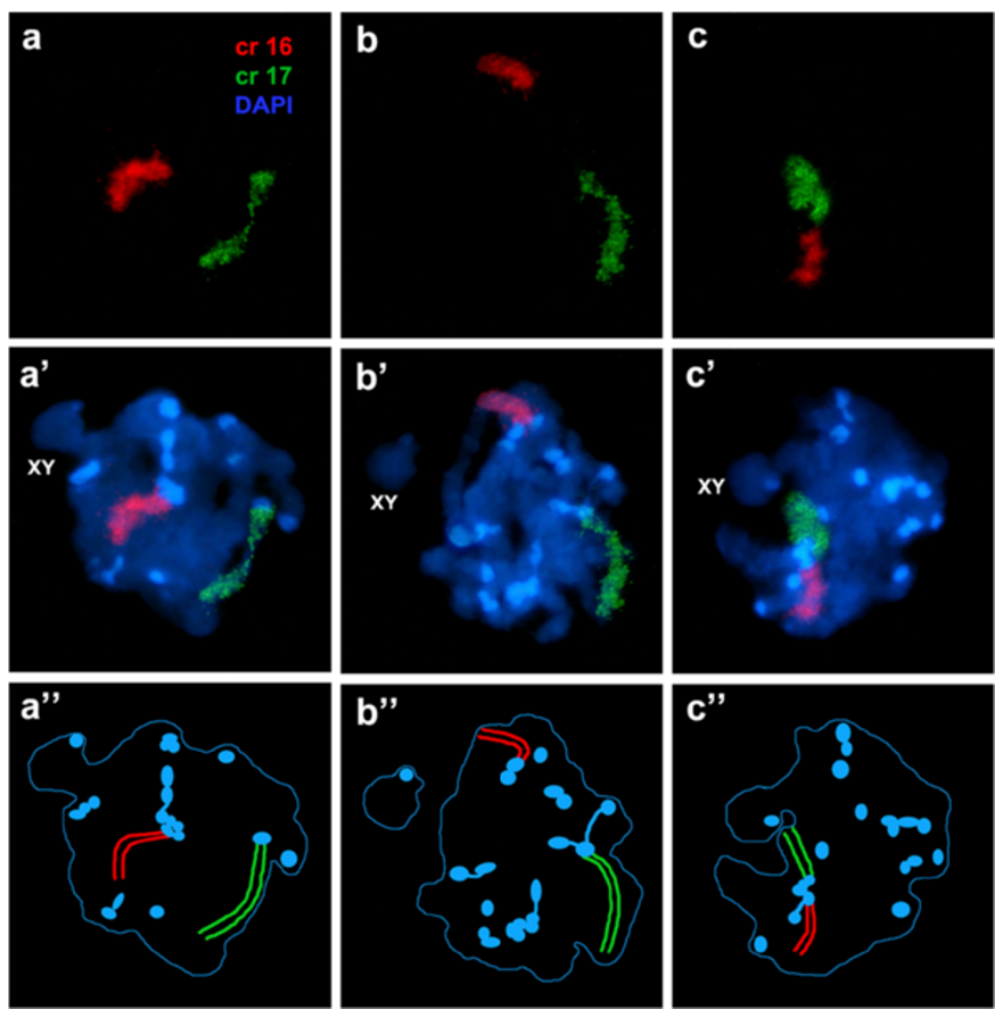

Figure 3 Associations between specific bivalents in pachytene nuclear squashs from $\mathbf{2 n}=\mathbf{4 0}$ spermatocytes. $a$, $b$, and $c$ : The bivalents 16 (red) and 17 (green) appear identified by FISH and specific probes. $\mathbf{a}^{\prime}, \mathbf{b}^{\prime}$ and $\mathbf{c}^{\prime}$ : Same nuclei counterstained with DAPI; $\mathbf{a}^{\prime \prime}, \mathbf{b}^{\mathbf{\prime}}$ and c': Representation of the painted bivalents 16 and 17 and the DAPI heterochromatin of the all bivalents. In the nucleus $a^{\prime \prime}$, the bivalent 16 through the heterochromatin is associated with 5 bivalents while the bivalent 17 is single; In the nucleus b", the bivalent 16 as the bivalent 17 are independently associated with other 2 bivalents; in the nucleus $c^{\prime \prime}$, both bivalents 16 and 17 are associated with each other and with two more bivalents through their heterochromatins.

elements, which included only 2 distinguishable bivalents, revealed that the frequency of events in which they can be associated with each other over all conditions was close to $12 \%$ (López-Fenner J, Berrios S, Manieu C, Page J, Fernández-Donoso R. Bivalent associations in Mus domesticus $2 n=40$ spermatocytes. Are they random? Submitted). If we use this theoretical value as a reference, we can consider that the observed frequencies of association between distinguishable elements could also

Table 1 Frequency of $\mathbf{2 n}=\mathbf{4 0}$ spermatocyte bearers with two given distinguished bivalents associated between them

\begin{tabular}{cc}
\hline $\begin{array}{c}\text { Pair of distinguishable } \\
\text { bivalents }\end{array}$ & $\begin{array}{c}\text { Spermatocyte percentage with those } \\
\text { bivalents associated between them }\end{array}$ \\
\hline 16 and 17 & 8 \\
9 and 14 & 10 \\
14 and 16 & 11 \\
9 and 17 & 10 \\
\hline
\end{tabular}

One hundred spermatocyte nuclei for each pair of bivalents were scored. The nuclei were stained with DAPI, and the specific bivalents were identified by FISH and specific DNA probes. be random, at least for the four pairs of bivalents here studied.

\section{Bivalent configuration and associations in $2 n=24$ pachytene spermatocytes}

In these nuclei only three SCs described regular arcs with the centromeres localized in one extreme near the nuclear envelope because only three autosomal bivalents are even telocentrics. The eight metacentric Rb bivalents formed longer SCs than those of the telocentric bivalents and the centromeres were localized in the middle of the metacentric $\mathrm{Rb}$ bivalents, far from the nuclear envelope. In a wellpreserved nucleus the focus was over one metacentric $\mathrm{Rb}$ bivalent (Figure 4a). In $2 n=24$ spermatocytes, associations were also produced through the pericentromeric heterochromatin, but mostly within groups of telocentric or metacentric bivalents (Figure 4b). Nuclear spreads with well-preserved clusters of heterochromatin showed two or more groups of metacentric bivalents associated in different number combinations and the telocentric bivalents mainly associated among themselves (Figure $4 c \& d$ ). The nuclear frequency per each chromosomal combination is 

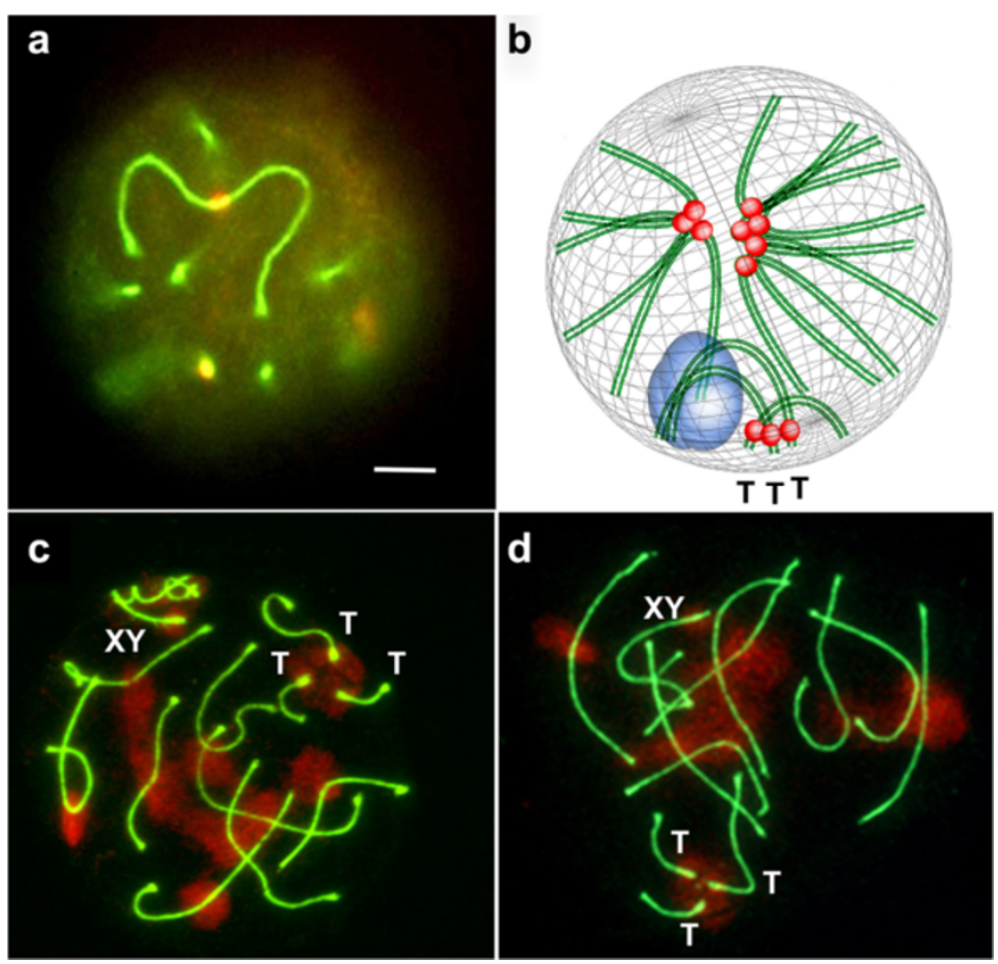

Figure $\mathbf{4}$ Nuclear architecture of spermatocytes $\mathbf{2 n}=\mathbf{2 4}$. a Distribution of metacentric and telocentric bivalents in a 3D pachytene nucleus from $2 n=24$ spermatocytes. The synaptonemal complexes (green) and centromeres (red) were identified by immunochemistry using anti-SYCP3 and anti-CENPA antibodies. The metacentric bivalents form SCs longer than those of the telocentric bivalents and the centromeres are localized in the middle of the metacentric bivalents unlike the terminal position in the telocentric bivalents. The focus is over one metacentric Rb bivalent. $\operatorname{Bar}=5 \mu \mathrm{m}$. b. Scheme representing the nuclear architecture of $2 n=24$ spermatocytes. The centromeric regions of the three telocentric bivalents (T) are located at the nuclear periphery and the centromeric regions of the metacentric bivalents at the nuclear center. The associations are produced mostly within groups of telocentric or metacentric bivalents. The XY chromatin is also respresented (blue). c \& $\mathbf{d}$ Associations between metacentric and telocentric bivalents through heterochromatin in $2 n=24$ spermatocytes. The SC (green) and the pericentromeric heterochromatin (red) were identified by immunochemistry using anti-SYCP3 and anti-H3K9me3 antibodies. The associations were mainly between the telocentric or the metacentric bivalents. c) Nucleus with 6 associated metacentric bivalents and 2 singles; the 3 telocentric bivalents appear all together. The $X Y$ bivalent is indicated. d) Nucleus with three groups of metacentric bivalents: 4 associated, 2 associated and 1 single; the 3 telocentric bivalents appear all together. The XY bivalent is indicated.

shown in Table 2. In 27\% of the spermatocytes the 3 telocentric bivalents were all together, in $56 \%$ of the nuclei only two bivalents were found associated and one single; and in approximately $17 \%$ of the nuclei the 3 telocentric bivalents were not in association, neither among themselves nor with other bivalents. Only $12 \%$ of the examined spermatocytes also showed associations between telocentric and metacentric bivalents (Table 2). In about 19\% of the spermatocytes some bivalents were associated with the XY bivalent.

\section{Trivalent configurations in spermatocyte nuclei of heterozygotes $2 n=32$}

In well-preserved nuclei of heterozygote spermatocytes, each trivalent showed three points of attachment to the nuclear envelope, two of which corresponded to the distal telomeres of the metacentric chromosomes synapsed with the telocentric chromosomes and the third given by the attachment of the proximal telomeres of the telocentric chromosomes. The third point of attachment to the nuclear envelope drags the pericentromeric heterochromatin of the 3 chromosomes involved in the trivalent towards the nuclear periphery. Throughout the meiotic prophase, the distance between the three telomere attachment points over the nuclear envelope increases, so that each trivalent may have different spatial configuration options. These configurations are observed in the spermatocyte shown in Figure $5 \mathrm{~b}$ and represented in the diagram shown in Figure 5a. In early prophase, most trivalents occupy a small space at the nuclear periphery, due to the close attachment on the nuclear envelope of the three telomeres. This configuration is modified in more advanced spermatocytes, where the distal telomeres appear attached at the opposite pole of the nuclear envelope with respect to the proximal telomere, so that the trivalent occupies almost the complete nuclear diameter. No associations between chromosomal axes were observed in any of the configurations in well-synapsed 
Table 2 Combination of associated and single metacentric/telocentric bivalents per nucleus observed in $2 n=24$ spermatocytes

\begin{tabular}{|c|c|c|c|c|}
\hline $\begin{array}{l}\text { Combinations between } \\
8 \text { metacentric bivalents }\end{array}$ & $\begin{array}{l}\text { Combinations between } \\
3 \text { telocentric bivalents }\end{array}$ & $\begin{array}{l}\text { Metacentric/Telocentric } \\
\text { associations }\end{array}$ & $\begin{array}{l}\text { Number of spermatocytes } \\
\text { per nuclear combination }\end{array}$ & $\begin{array}{c}\% \text { of spermatocytes } \\
\text { per Class }\end{array}$ \\
\hline $8-0$ & --- & & 0 & 0 \\
\hline $7-1$ & $2(3) ; 5(2-1)$ & & 7 & 7 \\
\hline $6-2$ & $3(3) ; 2(2-1)$ & & 5 & 12 \\
\hline $6-1-1$ & $2(3) ; 5(2-1)$ & $1(1 \mathrm{M} 2 \mathrm{~T})$ & 7 & \\
\hline $5-3$ & $1(1-1-1)$ & $1(3 \mathrm{M} 1 \mathrm{~T})$ & 1 & 8 \\
\hline $5-2-1$ & $1(3) ; 3(2-1)$ & & 4 & \\
\hline $5-1-1-1$ & $2(3) ; 1(1-1-1)$ & & 3 & \\
\hline $4-4$ & $1(2-1) ; 2(1-1-1)$ & & 3 & 31 \\
\hline $4-3-1$ & $3(3) ; 9(2-1) ; 1(1-1-1)$ & & 13 & \\
\hline $4-2-2$ & $2(3) ; 3(2-1) ; 1(1-1-1)$ & $1(2 \mathrm{M} 2 \mathrm{~T})$ & 6 & \\
\hline $4-2-1-1$ & $2(3) ; 4(2-1) ; 2(1-1-1)$ & $1(1 \mathrm{M} 1 \mathrm{~T})$ & 8 & \\
\hline $4-1-1-1-1$ & $1(1-1-1)$ & & 1 & \\
\hline $3-3-2$ & $1(3) ; 4(2-1) ; 2(1-1-1)$ & $1(1 \mathrm{M} 1 \mathrm{~T})$ & 7 & 38 \\
\hline $3-3-1-1$ & $3(3) ; 4(2-1) ; 3(1-1-1)$ & $1(3 \mathrm{M} 1 \mathrm{~T})$ & 10 & \\
\hline $3-2-2-1$ & $4(3) ; 8(2-1) ; 2(1-1-1)$ & $1(1 \mathrm{M} 1 \mathrm{~T})$ & 14 & \\
\hline $3-2-1-1-1$ & 1(3); 4(2-1); & $1(1 \mathrm{M} 1 \mathrm{~T})$ & 5 & \\
\hline $3-1-1-1-1-1$ & $2(2-1)$ & 1(3M1T) 1(1M1T) & 2 & \\
\hline $2-2-2-2$ & & & & 4 \\
\hline $2-2-2-1-1$ & 1(3): $2(2-1) ; 1(1-1-1)$ & 1(2M2T);1(1M1T) & 4 & \\
\hline \multicolumn{5}{|l|}{$2-2-1-1-1-1$} \\
\hline \multicolumn{5}{|l|}{$2-2-1-1-1-1$} \\
\hline \multicolumn{5}{|l|}{$2-1-1-1-1-1-1$} \\
\hline $1-1-1-1-1-1-1-1$ & & & & 0 \\
\hline TOTAL & $27(3) ; 56(2-1) ; 17(1-1-1)$ & 12 & 100 & 100 \\
\hline
\end{tabular}

100 nuclear microspreadings from $2 n=24$ spermatocytes were examined.

The SC and the pericentromeric heterochromatin were identified by immunochemistry using, respectively, anti-SYCP3 and anti-H3K9me3 antibodies (as in Figure 4c \& d). The first column shows the combination of associated and single metacentric bivalents per nucleus; The $2^{\text {nd }}$ column the combination of associated and single between 3 telocentric bivalents; the 3 th column the spermatocytes that showed associations between metacentric and telocentric bivalents; The $4^{\text {th }}$ column the frequency of spermatocytes per nuclear combination; and the $5^{\text {th }}$ column the frequency of spermatocytes per Class, considering as such, all the nuclei sharing the same highest combination of metacentric bivalent.

For example the third line of this Table means: In 5 spermatocytes the metacentrics were in a group of 6 and in another of two bivalents. In 3 of these spermatocytes the telocentrics were all together and in 2 they were in a combination 2 together and one alone. 12 spermatocytes were Class 6 or 12 had 6 as the higher number of associated metacentric bivalents.

trivalents. A different situation was observed on the trivalents in which the short arms of telocentric chromosomes remain asynapsed. These associations involved an endto-end junction between one or both single axes from two or more trivalents (Figure 5c) or between a trivalent single axis and the $\mathrm{X}$ chromosome single axis (Figure $5 \mathrm{~d}$ ). In both cases, as revealed by immunocytochemistry, the SYCP1 protein is detected at the end-to-end contact region (Figure $5 \mathrm{c} \& \mathrm{~d}$ ). The asynapsed trivalents also presented ectopic associations just mediated by heterocromatin from different trivalents or between a trivalent and the $\mathrm{XY}$ chromatin (Figure $5 \mathrm{e} \& \mathrm{f}$ ). In these situations the heterochromatin was modified as it can be demonstrated by the presence of the histone protein $\gamma \mathrm{H} 2 \mathrm{AX}$ (Figure $5 \mathrm{e} \& \mathrm{f}$ ). In $68 \%$ of mid pachytene, the single axes of partially-asynapsed trivalents established ectopic associations amongst themselves (15,7\%), with the XY bivalent $(27,1 \%)$ or both $(23,6 \%)$ (Table 3$)$. Only about $5 \%$ of the mid pachytene with well-synapsed trivalents presented ectopic associations, which were just mediated by heterocromatin from different trivalents or between a trivalent and the XY bivalent (Table 3). In late pachytene or diplotene spermatocytes, which mostly present just one asynapsed trivalent or the 8 trivalents completely synapsed, ectopic associations were rarely observed (not shown).

\section{Discussion}

The nuclear architecture of spermatocytes in meiotic prophase is primarily determined by the synaptic organization of the bivalents, bound by their telomeres to the nuclear 



Figure 5 Configurations of synapsed trivalents in pachytene nuclei of $2 n=32$ spermatocytes $(a-b)$ and ectopic associations between asynapsed trivalents and the $\mathbf{X Y}$ bivalent $(\mathbf{d}-\mathbf{f})$. $\mathbf{a}-\mathbf{b}$. Trivalent configurations in a diagram (a) and in a well preserved nucleus (b). The synaptonemal complexes (green) and centromeres (red) were identified by immunochemistry, using, respectively, anti-SYCP3 and anti-CENPA antibodies. The trivalents present 3 points of attachment to the nuclear envelope, two of which correspond to the distal telomeres of the metacentric chromosomes synapsed with the long arms of the telocentric chromosomes (arrowheads), and the third to the attachment of the proximal telomeres of the telocentric chromosomes (arrow). The third point drags the pericentromeric heterochromatin of the 3 chromosomes involved in the trivalent towards the nuclear periphery. c-d. In nuclear spreads the chromosomal axes (red) and central element of the SC (green) were identified by immunochemistry, using, respectively, anti-SYCP3 and anti-SYCP1 antibodies. c) Two asynapsed trivalents are associated through both single axes from the two telocentric short arms (arrow). In both bonding zones, the protein SYCP1 was detected (yellow). The 6-remaning trivalents are totally synapsed. d) The single axis of an asynapsed trivalent is bound to the $X$ chromosome single axis. At the end-to-end contact region, the SYCP1 protein was detected (arrowhead). e-f. $\mathrm{YH} 2 \mathrm{AX}$ antibodies intensely labels the XY chromatin (blue), the chromatin surrounding asynapsed trivalent AEs, and is absent in the chromatin of synapsed bivalents. e) One asynapsed trivalent is associated to the XY bivalent only through chromatin where the modified histone $\mathrm{YH} 2 \mathrm{AX}$ is detected (arrowhead). The 7-remaning trivalents are totally synapsed, not associated at all and without detection of $\mathrm{YH} 2 \mathrm{AX}$. f) Two asynapsed trivalents (arrows) and one asynapsed trivalent and the XY bivalent (arrowhead), are associated among them only through chromatin where the histone $\mathrm{YH} 2 \mathrm{AX}$ is detected.

envelope and describing arc-shaped trajectories through the nuclear space. However, over this basic meiotic organization, the spermatocyte nuclear architecture is also conditioned by the individual characteristics of the chromosomes and the opportunity for interactions between their domains. The homogeneity in the morphology of the chromosomes of Mus domesticus $2 \mathrm{n}=40$, in addition to the numerous subspecies with 
Table 3 Number of partially asynapsed trivalents and the type of ectopic asociations in $2 n=32$ heterocygote spermatocytes

\begin{tabular}{ccccccccccccc}
\hline & Asynapsed trivalents & $\mathbf{0}$ & $\mathbf{1}$ & $\mathbf{2}$ & $\mathbf{3}$ & $\mathbf{4}$ & $\mathbf{5}$ & $\mathbf{6}$ & $\mathbf{7}$ & $\mathbf{8}$ & Total & $\mathbf{\%}$ \\
\hline Ectopic associations & none & 35 & 1 & 3 & 4 & 3 & 2 & 0 & 0 & 0 & 47 & 33,6 \\
& XY/triv & 2 & 13 & 8 & 6 & 8 & 3 & 0 & 0 & 0 & 38 & 27,1 \\
& triv/triv & 0 & 0 & 5 & 7 & 4 & 4 & 0 & 0 & 0 & 22 & 15,7 \\
& both & 0 & 0 & 0 & 3 & 9 & 12 & 3 & 2 & 3 & 33 & 23,6 \\
& Total nuclei & 37 & 14 & 16 & 20 & 24 & 21 & 3 & 2 & 3 & 140 & 100 \\
\hline
\end{tabular}

140 nuclear microspreadings from $2 n=32$ pachytene spermatocytes were examined.

The single axes and central element of the SC were identified by immunochemistry, using, respectively, anti-SYCP3 and anti-SYCP1 antibodies (as in Figure 5c \& d).

reduced diploid numbers and carriers of metacentric $\mathrm{Rb}$ chromosomes, makes this species a convenient model to evaluate the above proposal. In this sense, the comparative analysis of the nuclear architecture of Mus spermatocytes with different chromosomal constitutions $(2 n=40$, $2 n=24$ and $2 n=32$ ) allowed us to establish which differences in nuclear organization are attributable to $\mathrm{Rb}$ chromosomes.

Moreover, all telocentric chromosomes of Mus $2 n=40$ exhibit abundant pericentromeric heterochromatin that is composed of two distinct repetitive DNA sequences, the minor and major satellites. It has been shown that the major satellites with the heterochromatin protein 1alpha form clusters, creating an association of several bivalents [21]. Previous analyses have allowed us to demonstrate that associations between autosomal bivalents mediated by heterochromatin are very frequent and can be considered as being random [6]. In this work, in which two bivalents were distinguishable each time, we found that the frequency of association between them was close to $10 \%$, coinciding with the expected rate if associations were at random. There were no significant differences in the frequency of association by spermatocyte between chromosome pairs that form $\mathrm{Rb}$ chromosomes (9/14 and 16/17) versus those that do not (9/16 and 14/17), nor did we find higher frequencies of association between bivalents 16 and 17 that are nucleolar and whose NOR regions are both near the pericentromeric heterochromatin. Ribosomal gene expression occurs in pachytene spermatocytes; this situation may have contributed to the association of NORs from different bivalents in the production of a common nucleolus, as has been described in somatic and meiotic cells $[5,13]$. However, as Mus has multiple nucleolar chromosomes, we cannot rule out preferential associations among other nucleolar chromosomes [22]. In any case, frequent random associations among all bivalents would be consistent with the necessary physical proximity among their heterochromatic regions for $\mathrm{Rb}$ fusions to occur among any of the 19 bivalents, as has been described for the different subspecies of Mus [18]. On the other hand, the homology of satDNA sequences shared by the mouse telocentric chromosomes [17] might have occurred by means of multiple small exchanges among these large tracts of tandemly repeated DNA [23]. The associations among the autosomal bivalents during meiotic prophase, precisely through their heterochromatic domains, would also provide a scenario in which these frequent recombinational exchanges between non-homologous chromosomes may have occurred. A similar origin has been proposed for the concerted evolution of the ribosomal DNA spacers on non-homologous nucleolar chromosomes [24-26].

In many organisms, a meiosis-specific organization of chromosomes called the "bouquet configuration," which is a clustering of telomeres on the inner nuclear envelope, appears to facilitate homologous recognition and alignment by concentrating all chromosomes within a limited region of the nuclear volume [27-30]. In the bouquet of the $2 n=40$ spermatocytes, all of the heterochromatic ends of the telocentric chromosomes cluster together, forming a large chromocenter. Later, the progress of the prophase increases the nuclear volume and the movement of telomeres over the nuclear envelope, and consequently the great original chromocenter breaks down into the smaller chromocenters seen in the pachytene stage [6]. In contrast, during the bouquet of $2 n=24$ spermatocytes, it was remarkable, that only two chromocenters were observed, a small one at the nuclear periphery and another with abundant heterochromatin toward the center of the nucleus. Therefore, two different areas for association are characteristic for these nuclei: one among the heterochromatins of telocentric bivalents, and another among the heterochromatins of metacentric bivalents. This organization continues toward the pachytene stage, where most of the spermatocytes show the telocentric bivalents tightly associated among themselves and the metacentric bivalents associated in a looser way forming two or more groups. We suggest that this new nuclear architecture would favor the progressive fusion of the remaining telocentric chromosomes. It is also possible that this narrowing of association opportunities is related to the overall trend toward production of metacentric chromosomes, as observed in the chromosomal evolution 
of this species [16,31]. On the other hand, association between the heterochromatic regions of the metacentric bivalents is the scenario in which WARTS may occur. In these $\mathrm{Rb}$ rearrangements, described also in the karyotypes of Mus, two $\mathrm{Rb}$ metacentric chromosomes exchange their arms due to breakage and reciprocal fusion at the pericentric heterochromatin level of both [32,33]. The described topological nuclear organization allows for the repeated encounter between chromosomal domains that may eventually experience rearrangements among them. However, under this topographic scenario it is also necessary to consider that during meiotic prophase, a programmed induction of DNA double-strand breaks (DSBs) leads to the exchange of genetic material between homologous chromosomes [34]. DSBs preferentially occurs at discrete sites called hotspots whose localization seems to be influenced by both local chromatin and higher-order chromosome structures [35]. It is not known if hotspots are preferentially localized at the heterochromatic domains of the Mus spermatocytes. However, on these meiotic prophase nuclei the full DNA repair machinery would be available, which could account for an exchange between heterologous DNA that may change the structure of the involved chromosomes.

In heterozygote $2 \mathrm{n}=32$ spermatocytes, another nuclear architecture emerges, which in this case accounts for the meeting of two different chromosome complements, one from $2 n=40$ homozygotes and the other from $2 \mathrm{n}=24$ homozygotes. These hybrids, or similar, may occur naturally in any overlapping area of two subpopulations of Mus, one bearing the ancestral karyotype and other that has diverged by the presence of multiple $\mathrm{Rb}$ chromosomes. In $\mathrm{Rb}$ heterozygote spermatocytes, recognition and synapsis between the two parental chromosome complements occur, forming eight trivalents, three telocentric bivalents, and a sexual bivalent, similar to what has been previously described for other Rb hybrids of Mus [36]. Each trivalent has three points of attachment to the nuclear envelope, two corresponding to the distal telomeres and the third to the heterologous proximal ends of the telocentric chromosomes. This organization leads to the three centromeres clustering together at the nuclear periphery. Depending on the distance between the distal and proximal telomeres the trivalents may take different configurations at the nuclear space. The ultimate reason why telomeres move away or why the trivalents present different configurations remains unknown. On the other hand, in any of these configurations, the centromeric and heterochromatic domains of the very same eight pairs of telocentric chromosomes are always together in every trivalent and spermatocyte. This repeated convergence of the same pairs of heterochromatic domains should be also decisive in favoring the formation of new metacentric $\mathrm{Rb}$ chromosomes.
It has been reported that approximately $80 \%$ of the heterozygote spermatocytes in early prophase shows 2 to 6 trivalents with varying degrees of asynapsis between the short arms of telocentric chromosomes [20]. Chromatin surrounding these areas of asynapsis experiences changes in condensation and modifications on the underlying proteins resulting in transcriptional repression. This entire phenomenon is known as MSUC for "Meiotic Silencing of Asynapsed Chromatin" [37]. Some of the asynapsed trivalents were only connected between them or with the XY bivalent through this kind of chromatin. It has been reported that MSUC also delay the progress of meiotic prophase, which gives the opportunity that these chromosomal regions reach the synapsis what apparently it happens in many of the spermatocytes [20]. Despite of this, we observed that in $68 \%$ of mid pachytene nuclei, the single axes of partially asynapsed trivalents established ectopic associations amongst themselves (15,7\%), with the XY bivalent $(27,1 \%)$ or both $(23,6 \%)$. Furthermore, the presence of SCP1 protein between the heterologous axes demonstrates the formation of the SC medial element and thus the possibility of recombination between heterologous chromosomal regions, which can later lead to segregational problems between those chromosomes. Therefore, the configuration of trivalents and the relationships among their unsynaptic axes impact globally the architecture of the heterozygote pachytene nuclei and possibly may have consequences for the survival of the spermatocytes. Thus, ectopic bonds could be quite deleterious to the normal progress of prophase and also to the subsequent segregation of the involved chromosomes. Spermatocytes carrying these ectopic joints may be eliminated by apoptosis in prophase or metaphase I, a phenomenon that has been observed in the Rb heterozygote male germ line $[38,39]$. All of these situations could explain the reduced fertility found in $\mathrm{Rb}$ hybrids [38,40-42].

Clearly, the multiple presence of $\mathrm{Rb}$ metacentric chromosomes in $2 n=24$ spermatocytes, as in the $2 n=32$ heterozygotes, determines a new organization and distribution of chromosomal domains in the meiotic prophase nucleus and consequently changes the possibilities for interchromosomal relationships as compared to those present in the $2 n=40$ spermatocytes. The nuclear architecture can achieve a new optimum as have been seen in homozygote spermatocytes $2 \mathrm{n}=24$. However, in heterozygote nuclei, the areas for meeting and interaction between chromosomal domains, which actually define the nuclear architecture, are unclear and their emerging trials rather seem to produce additional instability.

On the other hand, the significant changes in the spermatocyte nuclear architecture described here allow us to better understand the difficulties that a new $\mathrm{Rb}$ chromosomes face in surviving meiosis, and therefore to 
be inherited by the progeny and propagated into the reproductive community.

\section{Conclusions}

$\mathrm{The} \mathrm{Rb}$ chromosomes pose sharp restrictions for interactions in the $2 \mathrm{n}=24$ and $2 \mathrm{n}=32$ spermatocytes, as compared to the ample possibilities for interactions between bivalents in the $2 \mathrm{n}=40$ spermatocytes.

The emergence of $\mathrm{Rb}$ chromosomes changes the ancestral nuclear architecture of $2 \mathrm{n}=40$ spermatocytes since they establish new types of interactions among chromosomal domains.

The associations are produced through centromeric and heterochromatic regions at the nuclear periphery among telocentric bivalents and at the nuclear center among $\mathrm{Rb}$ metacentric ones.

\section{Methods}

We analyzed spermatocytes from four male threemonth-old Mus domesticus 2n $=40$ CD1 mice with all telocentric chromosomes; spermatocytes from four males of the Milano II $2 n=24$ with eight pairs of homozygote $\mathrm{Rb}$ metacentric chromosomes; and spermatocytes from four heterozygote $\mathrm{Rb}$ mice $2 \mathrm{n}=32$ with eight single $\mathrm{Rb}$ metacentric chromosomes. The heterozygote mice were generated by mating females of the laboratory strain CD1 $2 n=40$ and males of the Milano II race. Male and female specimens from the original natural populations were donated to our laboratory, by Drs Carlo Redi and Silvia Garagna from the Pavia University, Italy, as part of a collaborative research proyect.

The $\mathrm{Rb}$ chromosomes were the following: $\mathrm{Rb}(2.12)$, $\mathrm{Rb}$ (3.4), Rb (5.15), Rb (6.7), Rb (8.11), Rb (9.14), Rb (10.13), Rb (16.17). Numbers are according the $2 n=40$ standard karyotype.

Mice were maintained at $22^{\circ} \mathrm{C}$ with a light/dark cycle of 12/12 hours and fed ad libitum. Procedures involving the use of the mice were reviewed and approved by the Ethics Committee of the Faculty of Medicine, Universidad de Chile and by the Ethics Committee of the Chilean National Science Foundation FONDECYT-CONICYT.

\section{Spermatocyte squashes with 3-D preserved nuclei}

Spermatocyte squashes that preserved the nuclear volume were obtained following the procedure described by Page et al. [43]. Testes were removed and fixed in $2 \%$ formaldehyde in PBS containing 0.05\% Triton X-100. Pieces of tubules were placed in a drop of fixing solution on a slide. They were gently minced with tweezers, and then a coverslip was added. Exerting pressure on the coverslip squashed the cells. The slides were frozen in liquid nitrogen, and coverslips were then removed.

\section{Spermatocyte nuclear spreads}

Spermatocyte spreads were obtained following the procedure described by Peters et al. [44]. Briefly, a testicular cell suspension in $100 \mathrm{mM}$ sucrose was spread onto a slide dipped in $1 \%$ paraformaldehyde in distilled water containing $0.15 \%$ Triton X-100 then left to dry for two hours in a moist chamber. The slides were subsequently washed with $0.08 \%$ Photoflo (Kodak), air-dried, and rehydrated in PBS.

\section{Immunochemical identification of bivalents}

The slides were incubated for 45 minutes at $37^{\circ} \mathrm{C}$ in a moist chamber with the primary antibodies: rabbit antiSYCP3 1:100 (Abcam, ab15093); mouse anti-CENPA 1:200 (Abcam, ab13939); rabbit anti-H3K9me3 1:200 (Abcam ab8580); or mouse anti-phospho-histone H2AX (Ser139) 1:1000 clone JBW301 (Upstate, 05-636). Then, the slides were incubated for 30 minutes at room temperature with the secondary antibodies: FITC-conjugated goat antimouse IgG (1:50) (Sigma), or Texas red-conjugated goat anti rabbit IgG (1:200) (Jackson). Slides were counterstained with $1 \mu \mathrm{g} / \mathrm{ml}$ DAPI (4,6-diamidino-2-phenylindole). Finally, slides were rinsed in PBS and mounted in Vectashield (Vector).

\section{In situ hybridization}

In the microspreads of the $2 n=40$ spermatocytes, chromosomes 9, 14, 16, or 17 were identified by in situ hybridization using commercial probes (MetaSystems). The slides were treated for 5 minutes in 1XPBS, dehydrated in a series of $70,80,90$, and $100 \%$ ethanol for 2 minutes each, and air-dried at room temperature. The slides with the samples and the DNA probe were denatured together at $75^{\circ} \mathrm{C}$ for 2 minutes. Then, the slides were incubated in a humid chamber at $41^{\circ} \mathrm{C}$ for 16 hours. Next, the coverslip was removed, and the slides were washed in: 0.4X SSC at $72^{\circ} \mathrm{C}$ for 2 minutes; $2 \mathrm{XSSC}$ with $0.05 \%$ Tween 20 at room temperature for 30 seconds, and twice in $1 \times$ PBS for 5 minutes each. Nuclear contrast was performed with DAPI, and coverslips were mounted with Vectashield.

\section{Images}

Observations were made using a Nikon (Tokyo, Japan) Optiphot or Olympus BX61 microscope equipped with epifluorescence optics, and the images were photographed on a DS camera control unit DS-L1 Nikon or captured with an Olympus DP70 digital camera. All images were processed using Adobe Photoshop CS5.1 software or the public domain software Image ( $\mathrm{Na}$ tional Institutes of Health, United States; http://rsb. info.nih.gov/ij). 


\section{Competing interests}

The authors declare that they have no competing interests.

\section{Authors' contributions}

$\mathrm{SB}, J \mathrm{~L}$ drafted the manuscript and prepared the figures; $\mathrm{CM}$, carried out the FISH experiments and recorded the bivalent configurations; MG, JP, MM, carried out the nuclear spreadings and immunochemical experiments; EA, carried out the animal crosses and karyotypes; RF, represented the 3D nuclei and recorded the ectopic associations data. All authors read and approved the final manuscript.

\section{Acknowledgements}

FONDECYT Project \#1120160, Chile, and BFU 2009/10987 del Ministerio de Ciencia e Innovación, España, supported this work.

\section{Author details}

${ }^{1}$ Programa Genética Humana, ICBM, Facultad de Medicina, Universidad de Chile, Santiago, Chile. ${ }^{2}$ Departamento de Ingeniería Matemática, Universidad de La Frontera, Temuco, Chile. ${ }^{3}$ Departamento de Biología Celular, Universidad Autónoma de Madrid, Madrid, España.

Received: 18 April 2014 Accepted: 21 April 2014

Published: 14 May 2014

\section{References}

1. Zickler D, Kleckner N: Meiotic chromosomes: integrating structure and function. Annu Rev Genet 1999, 33:603-754.

2. Page SL, Hawley RS: The genetics and molecular biology of the synaptonemal complex. Annu Rev Cell Dev Biol 2004, 20:525-558.

3. Cohen PE, Pollack SE, Pollard JW: Genetic analysis of chromosome pairing, recombination, and cell cycle control during first meiotic prophase in mammals. Endocr Rev 2006, 27(4):398-426.

4. Berrios S, Fernández-Donoso R, Ayarza E, Paulos A, Moreno M: Non-random distribution of the pericentromeric heterochromatin in meiotic prophase nuclei of mammalian spermatocytes. Genetica 1999, 106:187-195.

5. Berrios S, Fernández-Donoso R, Pincheira J, Page J, Manterola M, Cerda MC: Number and nuclear localisation of nucleoli in mammalian spermatocytes. Genetica 2004, 121(3):219-228.

6. Berrios S, Manterola M, Prieto Z, López-Fenner J, Page J, Fernández-Donoso R: Model of chromosome associations in Mus domesticus spermatocytes. Biol Res 2010, 43(3):275-285.

7. Heyting C: Synaptonemal complexes: structure and function. Curr Opin Cell Biol 1996, 8:389-396.

8. Von Wettstein D, Rasmussen SW, Holm PB: The synaptonemal complex in genetic segregation. Annu Rev Genet 1984, 18:331-413.

9. Yang F, Wang PJ: The mammalian synaptonemal complex: a scaffold and beyond. Genome Dyn 2009, 5:69-80.

10. Fraune J, Schramm S, Alsheimer M, Benavente R: The mammalian synaptonemal complex: protein components, assembly and role in meiotic recombination. Exp Cell Res 2012, 318(12):1340-1346.

11. Lammers JHM, Offenberg HH, Van Aalderen M, Vink ACG, Dietrich AJJ, Heyting C: The gene encoding a major component of the lateral elements of synaptonemal complexes of the rat is related to X-linked lymphocyte-regulated genes. Mol Cell Biol 1994, 14:1137-1146.

12. Offenberg HH, Schalk JA, RI M, Van Aalderen M, Kester HA, Dietrich AJ, Heyting C: SCP2: a major protein component of the axial elements of synaptonemal complexes of the rat. Nucleic Acids Res 1998, 26:2572-2579.

13. Cerda M, Berrios S, Fernández-Donoso R, Garagna S, Redi C: Organization of complex nuclear domains in somatic mouse cells. Biol Cell 1999, 91:55-65.

14. Tsujimoto Y, Gorham J, Cossman J, Jaffe E, Croce C: The t(14;18) chromosome translocations involved in B-cell neoplasms result from mistakes in VDJ joining. Science 1985, 229:1390-1393.

15. Comings DE, Avelino E: DNA loss during Robertsonian fusion in studies of the tobacco mouse. Nat New Biol 1972, 237(76):199.

16. King M: Species Evolution: The Role of Chromosome Change. Cambridge, U.K.: Cambridge Univ. Press; 1993.

17. Garagna S, Marziliano N, Zuccotti M, Searle JB, Capanna E, Redi CA Pericentromeric organization at the fusion point of mouse Robertsonian translocation chromosomes. Proc Natl Acad Sci U S A 2001, 98(1):171-175.

18. Redi C, Capanna E: Robertsonian heterozygotes in the house mouse and the fate of their germ cells. New York, New York: AlanR. Liss Inc; 1988.
19. Britton-Davidian J, Catalan JDA Graca Ramalhinho M, Ganem G, Auffray JC, Capela R, Biscoito M, Searle JB, Da Luz Mathias M: Rapid chromosomal evolution in island mice. Nature 2000, 403:158.

20. Manterola M, Page J, Vasco C, Berríos S, Parra MT, Viera A, Rufas JS, Zuccotti M, Garagna S, Fernández-Donoso R: A high incidence of meiotic silencing of asynapsed chromatin is not associated with substantial pachytene loss in heterozygous male mice carrying multiple simple robertsonian translocations. PLoS Genet 2009, 5(8):e1000625. Epub 2009 Aug 28.

21. Guenatri M, Bailly D, Maison C, Almouzni G: Mouse centric and pericentric satellite repeats form distinct functional heterochromatin. J Cell Biol 2004, 166:493-505.

22. Rowe LB, Janaswami PM, Barter ME, Birkenmeier EH: Genetic mapping of $18 \mathrm{~S}$ ribosomal RNA-related loci to mouse chromosomes 5, 6, 9, 12, 17 , 18, 19, and X. Mamm Genome 1996, 7(12):886-889.

23. Kalitsis $P$, Griffiths $B$, Choo KH: Mouse telocentric sequences reveal a high rate of homogenization and possible role in Robertsonian translocation. Proc Natl Acad Sci U S A 2006, 103(23):8786-8791.

24. Arnheim N, Krystal M, Schmickel R, Wilson G, Ryder O, Zimmer E: Molecular evidence for genetic exchanges among ribosomal genes on nonhomologous chromosomes in man and apes. Proc Natl Acad Sci U S A 1980, 77(12):7323-7327.

25. Gonzalez IL, Sylvester JE: Human rDNA: evolutionary patterns within the genes and tandem arrays derived from multiple chromosomes. Genomics 2001, 73(3):255-263.

26. Eickbush $\mathrm{TH}$, Eickbush DG: Finely orchestrated movements: evolution of the ribosomal RNA genes. Genetics 2007, 175(2):477-485.

27. Scherthan H, Weich S, Schwegler H, Heyting C, Harle M, Cremer T: Centromere and telomere movements during early meiotic prophase of mouse and man are associated with the onset of chromosome pairing. J Cell Biol 1996, 134(5):1109-1125.

28. Scherthan H: A bouquet makes ends meet. Nat Rev Mol Cell Biol 2001 2:621-627

29. Carlton PM, Cande WZ: Telomeres act autonomously in maize to organize the meiotic bouquet from semipolarized chromosome orientation. J Cell Biol 2002, 157(2):231-242

30. Harper L, Golubovskaya I, Cande WZ: A bouquet of chromosomes. J Cell Sci 2004, 117:4025-4032.

31. Searle JB: Speciation, chromosomes, and genomes. Genome Res 1998, 8(1):1-3.

32. Capanna E, Redi CA: Whole-arm reciprocal translocation (WART) between Robertsonian chromosomes: finding of a Robertsonian heterozygous mouse with karyotype derived through WARTs. Chromosome Res 1995, 3(2):135-137.

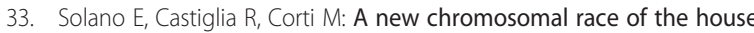
mouse, Mus musculus domesticus, in the Vulcano Island-Aeolian Archipelago, Italy. Hereditas 2007, 144(3):75-77.

34. Baudat $F$, Imai $Y$, de Massy B: Meiotic recombination in mammals: localization and regulation. Nat Rev Genet 2013, 14(11):794-806.

35. Yamada $\mathrm{T}$, Ohta $\mathrm{K}$ : Initiation of meiotic recombination in chromatin structure. J Biochem 2013, 154(2):107-114.

36. Grao P, Coll MD, Ponsà M, Egozcue J: Trivalent behavior during prophase I in male mice heterozygous for three Robertsonian translocations: an electron-microscopic study. Cytogenet Cell Genet 1989, 152(3-4):105-110.

37. Mahadevaiah SK, Costa Y, Turner JM: Using RNA FISH to study gene expression during mammalian meiosis. Methods Mol Biol 2009 558:433-444. Review.

38. Wallace BM, Searle JB, Everett CA: The effect of multiple simple Robertsonian heterozygosity on chromosome pairing and fertility of wild-stock house mice (Mus musculus domesticus). Cytogenet Genome Res 2002, 96(1-4):276-286.

39. Oka A, Mita A, Takada Y, Koseki H, Shiroishi T: Reproductive isolation in hybrid mice due to spermatogenesis defects at three meiotic stages. Genetics 2010, 186(1):339-351. Epub 2010 Jul 6.

40. Redi CA, Garagna S, Hilscher B, Winking H: The effects of some Robertsonian chromosome combinations on the seminiferous epithelium of the mouse. J Embryol Exp Morphol 1985, 85:1-19.

41. Hauffe HC, Searle JB: Chromosomal heterozygosity and fertility in house mice (Mus musculus domesticus) from Northern Italy. Genetics 1998, 150(3):1143-1154. 
42. Garagna S, Zuccotti M, Thornhill A, Fernandez-Donoso R, Berrios S, Capanna E, Redi CA: Alteration of nuclear architecture in male germ cells of chromosomally derived subfertile mice. J Cell Sci 2001, 114(Pt 24):4429-4434.

43. Page J, Suja JA, JI S, Rufas JS: Squash procedure for protein immunolocalization in meiotic cells. Chromosom Res 1998, 6:639-664.

44. Peters AH, Plug AW, Van Vugt MJ, De Boer P: A drying-down technique for the spreading of mammalian meiocytes from the male and female germline. Chromosome Res 1997, 5:66-68.

doi:10.1186/0717-6287-47-16

Cite this article as: Berríos et al:: Robertsonian chromosomes and the nuclear architecture of mouse meiotic prophase spermatocytes.

Biological Research 2014 47:16.

\section{Submit your next manuscript to BioMed Central and take full advantage of:}

- Convenient online submission

- Thorough peer review

- No space constraints or color figure charges

- Immediate publication on acceptance

- Inclusion in PubMed, CAS, Scopus and Google Scholar

- Research which is freely available for redistribution 